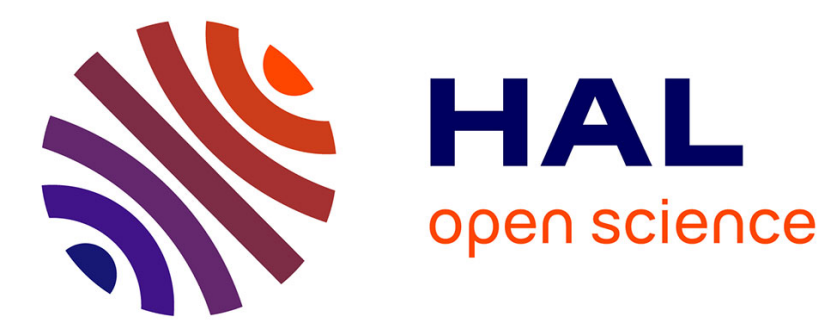

\title{
Sans l'imparfait, les vendanges tardives ne rentraient pas dans la jupe rhénane... Sur l'imparfait contrefactuel, pour avancer
}

Jacques Bres

\section{- To cite this version:}

Jacques Bres. Sans l'imparfait, les vendanges tardives ne rentraient pas dans la jupe rhénane... Sur l'imparfait contrefactuel, pour avancer. Syntaxe et Sémantique, 2009, 10, pp.33-50. hal-00438509

\section{HAL Id: hal-00438509 \\ https://hal.science/hal-00438509}

Submitted on 4 Jul 2018

HAL is a multi-disciplinary open access archive for the deposit and dissemination of scientific research documents, whether they are published or not. The documents may come from teaching and research institutions in France or abroad, or from public or private research centers.
L'archive ouverte pluridisciplinaire HAL, est destinée au dépôt et à la diffusion de documents scientifiques de niveau recherche, publiés ou non, émanant des établissements d'enseignement et de recherche français ou étrangers, des laboratoires publics ou privés. 
*2009, — «Sans l'imparfait, les vendanges tardives ne rentraient pas dans la jupe rhénane... Sur l'imparfait contrefactuel, pour avancer », Syntaxe et Sémantique 10, 33-50.

Jacques Bres

Praxiling UMR5267, CNRS-Montpellier III

\section{Sans l'imparfait, les vendanges tardives ne rentraient pas dans la jupe rhénane... Sur l'imparfait contrefactuel, pour avancer}

Le présent travaille dialogue avec l'article d'A.-M. Berthonneau et G. Kleiber, «Sur l'imparfait contrefactuel » (2006). Après avoir rappelé en cinq points les principaux acquis de la description du tour contrefactuel proposée par ces auteurs, on avance quelques éléments complémentaires. Ils concernent la liste des éléments frontaux qui peuvent participer à l'énoncé contrefactuel, la place et la fonction dudit énoncé, sa structure syntaxique, le rôle qu'y joue l’imparfait par rapport au conditionnel, les autres temps qui peuvent entrer dans sa production.

Mots-clefs : imparfait, contrefactualité, aspect, temps, interaction, contexte

En amical dialogue avec les travaux d'Anne-Marie Berthonneau et de Georges Kleiber sur l'imparfait

Sans l'imparfait, les vendanges tardives ne rentraient pas dans la jupe rhénane... Sur l'imparfait contrefactuel, pour avancer

(1) Pons comprit alors à quel saint dévouement, à quelle puissance d'amitié cette résurrection était due. " Sans toi, je mourais ! » dit-il en se sentant le visage doucement baigné par les larmes du bon Allemand, qui riait et qui pleurait tout à la fois. (Balzac, Le Cousin Pons)

Non, non, non, tout comme Saint Eloi, le brave cousin Pons n’est pas mort, car il parle encor... Comment l'imparfait, qui signifie d'habitude le factuel, peut-il actualiser ici un procès contrefactuel, ce qui se vérifie de sa possible glose par le conditionnel passé :

(1’) « Sans toi, je serais mort ! » dit-il [...]

Les linguistes ont déployé des trésors d'argumentation et y sont allés du joker de la valeur modale pour rendre compte de ce cygne noir... Sans Georges Kleiber et Anne-Marie Berthonneau (!), l'imparfait dans cet emploi était plus qu'un imparfait, ou moins qu'un imparfait... enfin n'était pas vraiment lui-même ${ }^{1}$. Leurs trois articles d'importance (2002,

\footnotetext{
${ }^{1}$ Exception faite de Wilmet 1997/2003. Je ne mentionne pas la liste des nombreux auteurs que cet emploi a interpellés : Kleiber et Berthonneau 2002 font de ces travaux antérieurs une présentation raisonnée et critique excellente.
} 
2003, 2006), ont apporté la preuve que l'imparfait contrefactuel était bien un imparfait comme tous les autres... c'est-à-dire notamment qu'il relevait d'un fonctionnement anaphorique méronomique. J'ai moi-même proposé une étude de cet emploi (Bres 2006) dans le cadre d'une approche aspectuelle de la temporalité verbale : j'y dialoguais alors avec les textes d'A.-M. Berthonneau et de G. Kleiber de 2002 et 2003, mais pas avec celui de 2006, encore non publié. Le présent travail entend poursuivre le dialogue anaphore - aspect ${ }^{2}$, en prenant en compte les avancées proposées dans Berthonneau et Kleiber 2006, pour tenter de faire cheminer le schmilblick sur le chemin descriptif et explicatif escarpé du tour contrefactuel à l'imparfait, qui dit à la fois que l'événement représenté par le procès n’a pas eu lieu et l'actualise comme s'il avait lieu...

Je commencerai, sans flagornerie aucune, par tirer un coup de chapeau à l'ensemble des travaux sur l'imparfait de G. Kleiber en solo, ou en tandem avec A.-M. Berthonneau : ils ont donné de la profondeur de champ à des emplois que la masse des réflexions déjà produites empêchaient de voir dans leur complexe simplicité, de sorte que des questions éculées comme l'imparfait forain, de rupture, de politesse, de concordance, etc... apparaissent, après le coup de torchon descriptif de nos auteurs, brillantes comme des sous neufs... Je le dis avec d'autant plus de liberté que je ne rentre pas dans la jupe explicative de l'anaphore méronomique qui y est développée (peut-être quelques kilos aspectualistes en trop !) : cette approche a donné du bon grain à moudre à l'approche aspectuelle dans les cadres de laquelle je me situe (Barceló et Bres 2006), en lui donnant l'occasion de sortir des exemples et des explications convenus dans lesquels elle ronronnait paisiblement...

Dans un premier temps, je ferai un bref rappel des principaux acquis de la description du tour contrefactuel proposée par A.-M. Berthonneau et G. Kleiber. Dans un second, j'entrerai en dialogue avec leurs analyses.

\section{Sans eux, le schmilblick de l’imparfait contrefactuel n'avançait guère...}

Les travaux de A.-M. Berthonneau et G. Kleiber ont considérablement enrichi la description de l'emploi contrefactuel de l'imparfait. Au risque d'une simplification quelque peu abusive, je résumerai leur apport en cinq points, les quatre premiers relevant de la description, le 5ème de l'explication :

(i) le tour n'est pas limité à un fait temporel d'addition d'une quantité minimale (un $x$ de plus, encore un peu, etc.) comme dans l'exemple prototypique qui nous vient de Guillaume 1929 :

(2) Un instant plus tard, le train déraillait

Il peut s’agir également de soustraction temporelle :

(3) - je suis en retard - Oh non, tu arrives au bon moment. Un peu plus tôt, c'était pas prêt (Berthonneau et Kleiber 2006 : 29)

Il peut également s'agir d'addition ou de soustraction d'une quantité autre que temporelle :

(4) Un père à son fils qui vient de grimper le Ventoux :

- Plus entraîné, tu le montais [le Ventoux] en même pas deux heures (oral conversationnel)

(5) Deux hommes parlent d'une ferme, flamboyante dans sa jeunesse :

- moins fardée, elle ressemblait à Monica Vitti (oral conversationnel)

\footnotetext{
${ }^{2}$ Précédemment : Kleiber 2003, 2006 ; Bres 2006a.
} 
Le tour peut également se construire en retranchant ou en ajoutant, non pas une quantité, mais un actant (1) ou un ingrédient d'une situation (6) :

(1) Pons comprit alors à quel saint dévouement, à quelle puissance d'amitié cette résurrection était due. "Sans toi, je mourais ! » dit-il en se sentant le visage [...]. (Balzac, Le Cousin Pons)

(6)[Nadine] ajouta : « Je meurs de faim.

- Évidemment tu n’as rien mangé. La morue était très bonne ; les fèves aussi.

- Une seule goutte d'huile et mon estomac débordait. » (Beauvoir de, Les Mandarins)

(ii) L’énoncé contrefactuel relève d'une «structure bipartite », ce qui le distingue radicalement de l'énoncé de rupture, de structure unaire, contrairement à l'analyse de Guillaume qui, pour les besoins de sa démonstration théorique, décrivait les deux tours comme similaires. Dans l'énoncé contrefactuel, le groupe initial $-x$ temps de plus, sans $x$, etc. - n'est pas un SP de phrase, mais un premier élément syntaxique, qui ne saurait être postposé sans que la contrefactualité ne s’efface :

(5’) elle ressemblait à Monica Vitti, moins fardée

(iii) Ce n'est pas l'imparfait qui est contrefactuel, ni forcément le circonstant, mais l'ensemble de la structure dans son rapport avec le co(n)texte). Le même énoncé « sans toi je m’ennuyais » est factuel en (7) et contrefactuel en (8) :

(7) Philippe lui demanda pourquoi elle n'avait fait cet appel de leur chambre. Inès répondit qu'elle y avait seulement pensé dans le hall.

- Nous devions pourtant nous retrouver là-haut, objecta Philippe.

- Oui, mais sans toi je m'ennuyais. Alors, je suis descendue.

- Justement, tu aurais pu passer le temps en téléphonant. (Droit, Le Retour)

(8) Pierre a rendu visite à Sophie, en convalescence. Le soir elle lui adresse le courriel suivant :

encore merci de ta visite, qui m'a fait grand plaisir. Sans toi cet aprem je m'ennuyais ferme... là au moins on a bien rigolé $[\ldots]$

Sans toi, Inès s’ennuyait effectivement ; Sophie, elle, se serait ennuyée...

Par-delà sa grande diversité de formes syntaxiques, le constituant frontal, pour entrer dans le tour contrefactuel, est soumis à une contrainte cotextuelle : il doit faire suite à un événement achevé passé, dont l'état final est connu, et se positionner anaphoriquement par rapport à lui en introduisant une modification.

(iv) Dans ce type d'énoncé, l'imparfait est toujours remplaçable par un conditionnel passé, alors que la réciproque n'est pas vraie : l'imparfait, pour entrer à titre d'ingrédient dans le tour contrefactuel, impose différentes contraintes (Berthonneau et Kleiber 2006 : 53-58, cf. infra 2.4.).

(v) Si l'imparfait peut participer à la production de la contrefactualité, il le doit à son fonctionnement anaphorique méronomique. Le procès à l'IMP exprime une autre fin que le dénouement factuel, qui en tant que fin possible constitue une partie du tout de l'événement.

\section{Encore quelques petits pas, et l'imparfait contrefactuel n'existait plus...}

En appui sur l'analyse d'occurrences attestées et dans le cadre d'une approche aspectuelle de la temporalité verbale, je propose de faire quelques pas supplémentaires - très précisément cinq - dans la description et l'explication du tour contrefactuel, certains qui viendront en confirmation de la piste développée, d'autres qui en proposeront un tracé sensiblement différent. 


\subsection{Plus longue, on n'en finissait pas...}

A.-M. Berthonneau et G. Kleiber ont considérablement allongé la liste des éléments frontaux qui peuvent participer à l'énoncé contrefactuel. Je propose à la fois de poursuivre cet allongement et... de le raccourcir quelque peu.

Les syntagmes pour un peu, n'était $\mathrm{x}$, sinon $^{3}$ peuvent entrer également dans la recette contrefactuelle :

(9) Un jour que le mage et sa bande étaient en train de festoyer chez nous, à nos dépens, le plafond de la salle à manger s'effondra. Pour un peu, il écrasait toute cette détestable société. Mais personne ne fut blessé. (Grenier R., Andrélie)

(10) Quand, à travers la fumée, se dressa la première flamme, nous fûmes pris de vraie démence et, n'était la chaleur qui nous en écartait, nous nous jetions tous ensemble, corps et âme, dans le brasier. (Zobel J., La Rue Cases-Nègres)

(11) il a fermé sa gueule sinon je le plantais (oral conversationnel)

Mais peut-être y a-t-il plus, et de plus d'importance : n'est-ce pas tout adjectif antéposé - et au-delà certains SP au fonctionnement similaire - qui est un possible candidat à la contrefactualité, pour peu que co(n)textuellement il se présente actualisant une qualité démentie par la réalité ? Soit (12) :

(12) Henri doit partir prochainement au Portugal sans sa maîtresse Paule: il prétexte un besoin de solitude...Mais il est forcé de lui apprendre qu'une autre jeune femme, Nadine, sera du voyage :

- $\quad$ [Nadine] va m'accompagner au Portugal.

- $\quad$ Non ! dit Paule. [...] Tu as dit que tu ne pouvais pas m’emmener.

- $\quad$ Tu n’y tenais pas, alors je ne me suis pas acharné.

- Je n’y tenais pas! Mais j'aurais donné une main pour aller avec toi ! Seulement j'ai compris que tu voulais être seul. Je veux bien me sacrifier à ta solitude, mais à Nadine, non !

- $\quad$ Seul ou avec Nadine, ça ne fait pas beaucoup de différence, dit-il avec mauvaise foi : puisque tu n'es pas jalouse d'elle.

- $\quad$ Ça fait toute la différence du monde ! dit-elle d'une voix bouleversée. Seul, j'étais avec toi, nous restions ensemble. Le premier voyage d'après-guerre : tu n'as pas le droit de le faire avec une autre. (Beauvoir de, Les Mandarins)

Autant dire que la liste des éléments pouvant jouer le rôle d'élément frontal du tour contrefactuel est loin d'être close...

En revanche, contrairement à toute la tradition qui remonte - au moins - à Guillaume 1929, et qu'A.-M. Berthonneau et G. Kleiber reconduisent et approfondissent dans la mesure où ils les décrivent finement (2006 : 42-46), je ne retiendrai peut-être pas les circonstants un x plus tard, un $x$ après, qui semblent pourtant prototypiques du tour, et ce pour des raisons d'attestation : la consultation de plusieurs centaines d'occurrences grâce à l'outil électronique (Frantext, Google) fait apparaître que dans tous les exemples, un x plus tard, et un $x$ après, lorsqu'ils sont suivis d'un imparfait, introduisent des procès factuels :

(13) Cette affiche le frappa, il s'arrêta pour la lire. Un instant après, il était dans le cul-de-sac de la Planchette, et il entrait au Plat d'étain, où était alors le bureau de la voiture de Lagny. (Hugo, Les Misérables)

(14) Assis sur mon lit, le dos contre un oreiller, je lisais. La porte d'entrée claqua, je relevai la tête. Un instant plus tard, Edmondsson apparaissait, le visage rayonnant. Elle voulait faire l'amour. (Toussaint, La Salle de bain)

\footnotetext{
${ }^{3}$ Et donc également sans quoi, faute de quoi, autrement, etc.
} 
Complémentairement, et qui semble confirmer ma réticence à l'endroit de un $x$ plus tard, et de un $x$ après : je n'ai pas relevé d'occurrence de ces tours suivis d'un conditionnel passé. Et si Guillaume, avec son exemple inventé Un instant plus tard, le train déraillait (1929 : 69), nous avait tous fait dérailler?

\subsection{Pour un peu, la fin contrefactuelle éclipsait la fin factuelle}

Selon A.-M. Berthonneau et G. Kleiber, «l'événement contrefactuel à l’imparfait apparaît comme étant une autre fin ou une autre issue possible de la situation [...]. La véritable fin (...) n’est jamais exprimée (...) car l'effet narratif de l'imparfait contrefactuel serait dégonflé par avance » (2006 : 36). Si effectivement ce peut être le cas, c'est loin de l'être toujours. La fin factuelle peut être explicitée après la fin contrefactuelle :

(15) Tout à coup ses yeux tombèrent sur les deux flambeaux d'argent que la réverbération faisait reluire vaguement sur la cheminée.

- Tiens ! pensa-t-il, tout Jean Valjean est encore là dedans. Il faut détruire cela.

Il prit les deux flambeaux.

Il y avait assez de feu pour qu'on pût les déformer assez promptement et en faire une sorte de lingot méconnaissable. [...] Il remua le brasier avec un des deux chandeliers.

Une minute de plus, et ils étaient dans le feu.

[long dialogue avec la voix de la conscience. Le lecteur ne sait si les chandeliers sont / ne sont pas dans le foyer, jusqu'à ce que, quelques 30 lignes plus avant :]

Il posa les flambeaux sur la cheminée. (Hugo, Les Misérables)

La fin contrefactuelle («Une minute de plus, et ils étaient dans le feu ») précède la fin factuelle, longuement différée ( «il posa les flambeaux sur la cheminée »). Il arrive même que la fin factuelle soit verbalisée avant la fin contrefactuelle :

(16) - On a gagné. On est tous là, entiers... Mais... Mais on est tous là, parce que ça [le feu] a fini juste à temps. Juste à temps : encore un peu et on y passait. Ça durait encore dix minutes, et j'étais mort, et les Bastides y passaient. Il s'en est fallu de peu. (Giono, Colline)

L’issue victorieuse de la lutte que les villageois ont menée contre le feu (« on est tous là, entiers ») précède la fin redoutée mais écartée ( « encore un peu et on y passait »).

Il me semble qu'il n'y a pas concurrence entre la fin factuelle et la fin contrefactuelle. Cette dernière joue un fort rôle d'évaluation, telle que définie par Labov (1972) : elle est là non pour se substituer à la fin factuelle mais pour souligner, par l'évocation d'une fin possible autre (le plus souvent plus dramatique), combien l'événement mis en récit est digne d'intérêt. Ce qui explique que l'énoncé contrefactuel puisse fonctionner en contexte non narratif, comme dans (5) :

(5) Deux hommes parlent d'une femme, flamboyante dans sa jeunesse :

- moins fardée, elle ressemblait à Monica Vitti

Hypothèse à vérifier : et si le tour contrefactuel, prototypiquement employé pour l'évaluation finale d'un récit ou d'une séquence narrative, avait vu son usage évaluatif élargi à tout autre type de discours : conversation comme en (5) ou discours publicitaire comme dans cette belle occurrence relevée par A.-M. Berthonneau et G. Kleiber :

(17) Moins cher, c’était du fil de fer (Leroy Merlin)

2.3. Encore un peu, et le tour contrefactuel était un tour hypothétique comme les autres. Subordination et corrélation 
A.-M. Berthonneau et G. Kleiber rapprochent le tour contrefactuel du tour hypothétique en si $P$, dans sa version d'irréel du passé, par lequel il peut être paraphrasé :

(18) Sans candidat MDC et PRG, l'issue du premier tour était différente, et la face de l'élection présidentielle était changée. (L. Jospin, Etre utile, Monde $1^{\mathrm{er}}$ février 2003)

(18') S'il n'y avait pas eu de candidat MDC et PRG, l'issue du premier tour (...)

J'ajouterai que, dans les tours hypothétiques à valeur d’irréel du passé, le second élément l'apodose - peut actualiser le procès au conditionnel passé, mais également à l'imparfait (19), comme dans le tour contrefactuel :

(19) commentaire télé en direct d'une étape du Tour de France 2008. Menchov, qui n'est qu'à quelques secondes de Evans au classement général, vient de creuser, dans l'ultime "bosse », un petit écart mais ensuite a été rattrapé :

- s'il avait pas coincé Menchov, il allait chercher Evans (juillet 2008)

A.-M. Berthonneau et G. Kleiber insistent cependant sur le fait que cela «ne signifie nullement que le constituant frontal soit à analyser lui-même comme une proposition hypothétique elliptique » (2006 : 33). Ce qu'ils appuient par deux faits : (i) dans les tours contrefactuels, on peut trouver ou ajouter le coordonnant et entre les deux éléments, alors que ce n'est pas possible entre la subordonnée en si $P$ et la principale :

(16) encore un peu $\boldsymbol{e t}$ on y passait. (Giono, Colline)

(16’) *si cela avait duré encore un peu, et on y passait.

(ii) la postposition, impossible avec le tour contrefactuel, est possible avec si $P$ :

(16') ?on y passait, encore un peu.

(16",') on y passait, si cela avait duré encore un peu.

Ce que vérifie doublement mon corpus : je ne dispose d'aucune occurrence de contrefactuel avec postposition du circonstant frontal ${ }^{4}$; alors que la postposition de si $P$ se voit attestée :

(21) Je perdais tout mon sang, et j'étais un homme mort si notre charrette ne se fût arrêtée devant une chaumière (Diderot, Jacques le Fataliste)

Ces deux arguments ne me paraissent cependant pas dirimants : le premier - l'impossibilité du et entre la subordonnée en si $P$ et la principale - vaut également pour certains tours typiquement contrefactuels, notamment ceux introduits pas sans :

(7) Sans toi cet aprem je m'ennuyais ferme

(7’) * Sans toi cet aprem et je m'ennuyais ferme

Le second - l'impossibilité de la postposition du circontant frontal dans le tour contrefactuel alors que la postposition de si $P$ est parfaitement réalisable - s'il correspond à la réalité des

${ }^{4}$ Lorsque le circonstant $x$ temps plus tard est postposé, c’est le sens factuel qui est actualisé :

(20) En ce moment, le vieillard disparut sous les flots. Oswald n'hésita plus, et s'élança dans la mer, malgré les vagues qui recouvraient sa tête. Il lutta cependant heureusement contre elles, atteignit le vieillard qui périssait un instant plus tard, le saisit et le ramena sur la rive. (Mme de Stael, Corinne ou l'Italie)

Le cotexte ultérieur explicite que ledit vieillard est mort et bien mort. Mais ce type d'occurrence n'est pas vraiment probant dans la mesure où je ne dispose pas d'occurrence où la frontalité de $x$ temps plus tard engendre l'effet de sens contrefactuel (cf. supra, 2.1.). 
faits, ne me semble pas être un argument en faveur de la différence irréductible des tours contrefactuel et hypothétique, mais tenir à ce que se voient comparés des éléments dissemblables syntaxiquement, ce que je vais expliciter.

Le tour hypothétique en si $P$ relève de la subordination ${ }^{5}$ (si $P, Q$ ). Le tour contrefactuel est analysé par A.-M. Berthonneau et G. Kleiber comme une «structure bipartite ». Ne peut-on aller plus loin dans la description et le caractériser comme une structure de corrélation? Corrélation que l’on trouve p. ex. dans les énoncés :

(22) Nourris un corbeau, il te crèvera les yeux

(23) Plus ça devient vieux, plus ça devient bête (J. Brel, Les Bourgeois)

qui, comme le tour contrefactuel, admettent tendanciellement le coordonnant et entre les deux segments (22') et n'admettent pas la postposition du premier segment (22') :

(22') Nourris un corbeau, et il te crèvera les yeux

$(22$ '”) *il te crèvera les yeux, nourris un corbeau

Rappelons que la relation d'hypothèse peut également se réaliser sous forme corrélative, avec dans l'apodose le conditionnel passé (24) mais également l'imparfait (25) :

(24) J'aurais été un peu ivrogne, dès mes débuts, je me serais aperçu de rien. (Céline, D’un château l'autre)

(25) Eussé-je été lu, je tentais de plaire, je redevenais merveilleux. Clandestin, je fus vrai. (Sartre, Les Mots).

Et il me semble que le rapprochement des tours contrefactuel et hypothétique est plus pertinent si on le fait avec une structure corrélative :

(7) moins fardée, elle ressemblait à Monica Vitti $\approx$ elle aurait été moins fardée, elle ressemblait à Monica Vitti

C'est d'ailleurs un exemple de ce type que l'occurrence (16), immédiatement après le tour contrefactuel (« encore un peu et on y passait ») réalise :

(16) Mais on est tous là, parce que ça [le feu] a fini juste à temps. Juste à temps : encore un peu et on y passait. Ça durait encore dix minutes, et j'étais mort, et les Bastides y passaient. Il s'en est fallu de peu. (Giono, Colline)

La protase conditionnante «ça durait encore dix minutes » est suivie d'une première apodose introduite par et («et j'étais mort »), elle-même suivie d'une seconde apodose coordonnée ( et les Bastides y passaient»). Et comme dans les tours contrefactuels prototypiques, la postposition de la protase est impossible :

(16’) *j'étais mort, et les Bastides y passaient, ça durait encore dix minutes.

Le tour contrefactuel corrélatif ressemble comme deux gouttes d'eau au tour hypothétique corrélatif. Mais a-t-on affaire à deux gouttes d'eau jumelles ou à une seule et même identité, sous deux vêtures à peine différentes ? Le tour contrefactuel ne relève-t-il pas pleinement de l'hypothèse par corrélation, sa seule spécificité tenant à ce que sa protase est elliptique (du sujet et du verbe notamment) ? Soit donc, non sans quelque provocation : le tour contrefactuel n’existe pas, nous l'avons déjà rencontré... dans le tour hypothétique.

\footnotetext{
${ }^{5}$ Ce qui explique l’impossibilité d'ajouter le coordonnant et entre les deux propositions.
} 


\subsection{L'imparfait en moins, la délicieuse petite frayeur de l'énoncé contrefactuel disparaissait. Du conditionnel et de l'imparfait}

Dans l'article de 2006, A.-M. Berthonneau et G. Kleiber explicitent à quoi tient la différence d'emploi du conditionnel passé et de l'imparfait dans le tour contrefactuel. Ils montrent que, dans ce type de contexte, si tout IMP peut être remplacé par un conditionnel passé, l'inverse n'est pas vrai, ce qui s'expliquerait par le fait que le conditionnel passé "possède intrinsèquement la valeur modale de contrefactualité » (2006: 54), mais non l'imparfait. Illustrons par des exemples qui prennent sans $x$ comme matériel : à l'imparfait de (26), je peux substituer le conditionnel passé sans restriction aucune :

(26) En furetant avec promptitude, je rencontrais fort heureusement un bras qui ne tenait à rien, le bras d'un Hercule ! un bon os auquel je dus mon salut. Sans ce secours inespéré, je périssais. (Balzac, Le Colonel Chabert, p.78)

(26’) Sans ce secours inespéré, j’aurais péri.

Par contre, la substitution de l'imparfait au conditionnel, parfois possible comme en (27), ne l'est pas en (28) ni en (29) :

(27) Avez-vous réussi à dîner le jour de mon départ? Je vous ai fait faire une journée de corvée, mais sans vous je ne serais pas venue à bout de partir. Avez-vous eu la bonté de ranger tout chez moi, de mettre dehors mes chambrières, de fermer portes et fenêtres, etc..? (Sand, Correspondance, 1833)

(27’) Je vous ai fait faire une journée de corvée, mais sans vous je ne venais pas à bout de partir.

(28) Il raconta comment il serait mort le 15 mai, sans le dévouement d'un garde national (L'Education sentimentale)

(28’) *Il raconta comment il était mort le 15 mai, sans le dévouement d'un garde national

(29) je t'aimais inconstant, qu'aurais-je fait fidèle? (Racine, Andromaque)

(29') *je t'aimais inconstant, que faisais-je fidèle?

L’imparfait impose différentes contraintes qui se voient pertinemment décrites (2006: 52 et sq.) : (i) l'imparfait a besoin du complément frontal, non le conditionnel (cas de (28)-(29)) ; (ii) il est impossible de modaliser la contrefactualité à l'imparfait ; (iii) si des précisions sur le procès sont fournies, on glisse vers un imparfait factuel ; (iv) l'imparfait doit apparaître comme étant la fin de l'épisode ; (v) il demande une situation antécédente déjà saillante.

Lorsque ces contraintes sont respectées, l'imparfait peut être un ingrédient du tour contrefactuel, du fait de son fonctionnement anaphorique méronomique :

Nous avons toujours affaire à une situation dans le passé dont le structure bipartite complément frontal + procès à l'imparfait exprime une fin ou une issue contrefactuelle. Le tout est la situation saillante nécessaire pour que l'on ait ce type d'emploi contrefactuel et le procès exprimé à l'imparfait est une partie de ce tout, puisqu'il s'agit d’une autre fin ou clôture ou résultat que la fin réelle ou résultat factuel. (2006 : 60)

La démonstration est parfaitement cohérente ${ }^{6}$ : l’imparfait n’a rien de modal, « il reste bien le même en fonctionnant comme un anaphorique méronomique » (2006 : 61). Pour autant, si se trouve bien décrite la différence de fonctionnement de l'imparfait et du conditionnel passé dans le tour contrefactuel, il me semble que reste inexpliquée la raison pour laquelle ce temps, par rapport au conditionnel, « augmente la dramatisation du récit » (Riegel et al. 1994 : 309), argument traditionnel des grammaires que A.-M. Berthonneau et G. Kleiber font leur.

A quoi tient « l'expressivité plus grande du tour à l'imparfait » (2006 : 53)? Cette question me semble d'importance parce que dans sa réponse réside peut-être la clé de l’emploi de

\footnotetext{
${ }^{6}$ A la réserve près mentionnée supra en 2.2. selon laquelle on n’a pas toujours un récit et son autre fin.
} 
l'imparfait en contexte contrefactuel. Je propose une hypothèse explicative dans les cadres d'une approche aspectuelle des temps verbaux (Bres 2005, Barceló et Bres 2006) qui se voient définis par des instructions temporelles et aspectuelles. Soit pour le conditionnel passé et l'imparfait :

\begin{tabular}{lllll} 
& & instruction temporelle & \multicolumn{2}{c}{ instructions aspectuelles } \\
COND. passé & il aurait plu & {$[+$ passé $][+$ ultérieur $]$} & {$[+$ extension $]$} & {$[ \pm$ incidence $]$} \\
IMP & il pleuvait & {$[+$ passé $]$} & {$[+$ tension $]$} & {$[-$ incidence $]$}
\end{tabular}

Ces instructions sont analysées comme l'offre des temps verbaux, offre qui lors de l'actualisation entre en interaction avec la demande contextuelle. L'interaction entre demande et offre peut être concordante ou tendanciellement discordante (Bres 2008). Dans le cas de l'énoncé contrefactuel, l'interaction est (i) concordante avec le conditionnel passé ; et (ii) tendanciellement discordante avec l'imparfait, ce qui va être brièvement exposé.

Commençons par définir la contrefactualité. Elle consiste à ouvrir une perspective dans le passé, et à déclarer que cette perspective n’a pas abouti à la réalisation du procès ; et, par inférence contextuelle, que ce procès non réalisé est différent de celui qui s’est effectivement réalisé. Un contexte contrefactuel demandera donc que le procès de l'énoncé soit (i) temporellement, situé dans le passé et en perspective ; et (ii) aspectuellement, que son temps impliqué soit représenté en extension : la saisie du procès à partir de sa borne terminale permet, lorsque la réalisation possible du procès est située dans le passé, de signifier que la perspective n'a plus d'avenir et qu'elle a pu ne pas se réaliser.

Comment les deux temps du conditionnel passé et de l'imparfait réagissent-ils à ces exigences?

(i) Le conditionnel passé ne me semble pas «intrinsèquement contrefactuel » (Berthonneau et Kleiber 2006 : 58) :

(30) Attends encore un peu... Mireille m'a dit qu'elle serait revenue à 7 h... elle va arriver (conversation, à 18h45)

(31) DSK et sa subordonnée auraient échangé des courriels intimes, avant de concrétiser leur relation lors d'une conférence en Europe. (Libération, 20 octobre 2008)

Aucune contrefactualité dans les procès de ces deux occurrences : Mireille en (30) sera peutêtre revenue à 19h ; DSK en (31) a bien peut-être échangé des messages et même concrétisé cet « accident du travail »...

Le conditionnel, présent comme passé, ne situe pas le procès lui-même mais un énonciateur dans le passé du locuteur (morphème -ai), à partir duquel est ouverte la perspective ultérieure (morphème -r) de réalisation d’un événement (Bres 2009). Lorsque cet événement est situé contextuellement dans le passé du locuteur, l'aspect extensif, en interaction avec cette situation temporelle, peut marquer l'irréel du passé (ce que ne ferait pas le conditionnel présent). L'offre aspectuo-temporelle du conditionnel passé est donc en interaction parfaitement concordante avec la demande cotextuelle de contrefactualité :

$\begin{array}{lll} & \text { instruction temporelle } & \text { instruction aspectuelle } \\ \begin{array}{lll}\text { demande de la contrefactualité } \\ \text { offre du conditionnel passé }\end{array} & \text { passé, ultériorité } & \text { extension } \\ \text { passé, ultériorité } & \text { extension, } \pm \text { incidence }\end{array}$

Les instructions du conditionnel passé saturent la demande contextuelle. Ce temps est la forme orthonymiquement attendue : 
(32) Dire que je l'ai connue confectionneuse de lingerie! Sans moi, plus de vingt fois elle serait tombée dans la crotte. Mais je l'y plongerai ! (L'Education sentimentale)

Ajoutons que c'est parce que l'interaction entre demande du contexte contrefactuel et offre du conditionnel passé est parfaitement concordante que, dans ce tour, l'imparfait peut toujours être remplacé par un conditionnel passé.

(ii) L’imparfait, s’il s'accorde avec la demande temporelle d'époque passée, est en discordance aspectuelle avec elle: à la demande d'ultériorité et d'extension de la contrefactualité, l’imparfait répond par l'offre de tension et de non-incidence :

instruction temporelle

passé, ultériorité

passé instruction aspectuelle

extension,

tension, - incidence

offre de l'imparfait

L’imparfait ne répond ni à la demande d'ouvrir une perspective ni à celle de déclarer cette perspective comme sans avenir: il ne dispose pas dans sa morphologie du $-r$; et offre l'instruction [+ tension] et non l'instruction [+ extension]. L'absence de $-r$ dans la morphologie de l'imparfait rend compte de ce que, dans certaines occurrences (supra (7) et (8)), il puisse y avoir ambiguïté entre les interprétations factuelle et contrefactuelle : l'imparfait, en n'ouvrant pas de perspective future, réfère habituellement à un fait dont la réalisation est représentée comme effectivement en cours dans le passé, et non pas envisagée comme pouvant se produire (et donc comme pouvant ne pas se produire) à un moment ultérieur. Mais en quoi la non-réalisation de la perspective ultérieure n'est-elle pas rédhibitoire, à savoir n'entraîne-t-elle pas l'impossibilité d'associer l'imparfait à la contrefactualité ? Le fait que ni le passé simple ni le passé composé ne peuvent entrer dans la recette contrefactuelle nous met sur la voie. La raison de cette impossibilité est selon moi aspectuelle $^{7}$ : le passé simple (par son instruction [+ incidence]), le passé composé (par son instruction [+ extension]) présupposent que le procès a atteint sa borne terminale (de l'intérieur pour le premier, de l'extérieur pour le second, Guillaume 1971 : 171, Leçon du 24 février 1949). Le procès est actualisé comme parfaitement accompli, puisque sa borne terminale est représentée comme atteinte. Ainsi «factualisé » dans le passé, le procès au passé simple comme au passé composé ne saurait entrer dans la combine contrefactuelle.

Le paramètre aspectuel, qui rend compte de l'impossibilité de ces temps, est également le principe explicatif de l'association de l'imparfait à la contrefactualité : de par son trait [incidence], l'imparfait saisit le temps interne du procès au-delà de sa borne initiale et en-deçà de sa borne terminale. Pour ne pas atteindre cette dernière, il l'inscrit de la sorte bien moins en réalité. Si le contexte le demande, il permettra, en légère dissonance, d'actualiser un procès contrefactuel. Non qu'il ait lui-même viré casaque : il n'est pas devenu contrefactuel par l'amicale pression du contexte. C'est sa formule aspectuelle qui autorise la production de cet effet de sens. Et la meilleure preuve qu'il est resté pleinement lui-même, c'est que l'effet de sens de supplément d'âme expressif, ou dramatique que les grammaires ${ }^{8}$ lui accordent par rapport au conditionnel passé procède directement des traits aspectuels de sa valeur en langue: il donne à voir le procès, contextuellement déclaré contrefactuel, dans le cours (possible) de sa réalisation passée ; il actualise un procès qui n’a pas eu lieu comme s’il avait lieu... A la différence du conditionnel passé, pleinement concordant avec la demande contextuelle de contrefactualité, l’imparfait permet de jouer à se faire une brève petite joie ou

\footnotetext{
7 Berthonneau et Kleiber 2006 expliquent l’impossibilité du passé simple et du passé composé dans le tour contrefactuel par le fait que ces temps ne fonctionnent pas selon l'anaphore méronomique.

${ }^{8}$ « Par une sorte de projection visionnaire, un procès avorté est imaginé dans son déroulement effectif » (Wilmet 1997/2003 : 424).
} 
une brève petite peur, selon que le procès contrefactuel est plus ou moins heureux que ce qui s'est effectivement passé :

(33) deux kilos de moins, je rentrais dans ma jupe (cité par Berthonneau et Kleiber)

(34) Le père, sans s'arrêter de planter les piquets, de tasser ses pierres, grognait :

- Quinze jours de plus, et la vigne davallait à la rivière ; après, il faut cent ans pour la remonter ! (Chabrol, Les Fous de Dieu)

Sans l'imparfait, les vendanges tardives de la vigne ne rentraient pas dans la jupe rhénane... Ce temps permet d'évoquer le passé non comme différent de ce qu'il a été (ce que fait le conditionnel passé), mais d'actualiser cette différence contrefactuelle comme si elle était factuelle...

\subsection{Encore quelques temps, et le tour contrefactuel livrait peut-être tous ses secrets...}

Avant de terminer, je voudrais juste pointer que la contrefactualité n'est pas la chasse gardée de l'imparfait. Viennent également fréquenter ce lieu, l'imparfait prospectif (35), le plus-queparfait (36) ${ }^{9}$, le présent (37)...

(35) Je peux dire que j'ai jamais entendu quelqu'un d'aussi furax. J'en revenais pas qu'un bonhomme si classe puisse dégoiser autant d'injures. Pour un peu c'est son téléphone, lui, qu'il allait bouffer. J'ai tenu bon jusqu'au bout dans la tempête, cramponnée à mon bigo comme un naufragé à sa bouée. (Seguin, L'Arme à gauche)

(36) Montparnasse lui-même, qui était peut-être un peu le gendre de Thénardier, cédait. Un moment de plus, ils étaient partis. Thénardier haletait sur son mur comme les naufragés de la Méduse sur leur radeau en voyant le navire apparu s'évanouir à l'horizon. (Hugo, Les Misérables, corpus A. lauze)

(37) il le tenait à la gorge tu vois comme ça et il serrait il serrait / encore un peu et il l'étrangle mais la prof est rentrée il s'est calmé il s'est assis tu vois et elle elle a fait comme si elle avait rien vu (oral conversationnel)

Reste à faire l'étude précise du fonctionnement de la contrefactualité avec ces formes temporelles.

\section{Sans cette conclusion, le coup de grâce n’était pas donné au tour contrefactuel...}

Après avoir rappelé les avancées dans la description et l'explication de l'imparfait dit contrefactuel réalisées par les travaux d'A.-M. Berthonneau et G. Kleiber, j’ai proposé de faire quelques petits pas pour progresser dans la description du tour contrefactuel : la liste des éléments frontaux susceptibles de rentrer dans la recette doit être allongée, et... peut-être raccourcie ; l'énoncé contrefactuel fonctionne comme une évaluation qui peut intervenir non seulement en récit, mais également dans d'autres genres du discours ; l'énoncé contrefactuel est un énoncé hypothétique corrélatif comme les autres, à l'ellipse près que réalise sa protase ; la plus-value qu'apporte l'imparfait par rapport au conditionnel passé tient à ce que l'interaction entre demande contextuelle et offre aspectuelle, concordante avec le conditionnel passé, est discordante avec l'imparfait qui donne à voir le procès, contextuellement posé ou présupposé comme n'ayant pas eu lieu...comme s'il avait lieu.

Une dernière remarque qui me semble porter le coup de grâce au tour contrefactuel en tant que phénomène linguistique spécifique : toutes les analyses, et la mienne jusqu’à ce point, s’accordent à décrire l'énoncé contrefactuel comme portant sur un événement passé. C’est

\footnotetext{
${ }^{9}$ Ce qui permet au plus-que-parfait d’être associé au contrefactuel alors qu'il est, comme le passé composé, une forme extensive, c'est son instruction [- incidence].
} 
effectivement le plus souvent le cas, mais pas toujours, comme un lecteur attentif n'aura pas manqué de le noter à propos de l'occurrence (12) que je rappelle (en abrégeant sa contextualisation) :

(12) Henri doit partir prochainement au Portugal sans sa maîtresse Paule: il prétexte un besoin de solitude...Mais il est forcé de lui apprendre qu'une autre jeune femme, Nadine, est du voyage :

- $\quad$ Seul ou avec Nadine, ça ne fait pas beaucoup de différence, dit-il avec mauvaise foi : puisque tu n’es pas jalouse d'elle.

- Ça fait toute la différence du monde ! dit-elle d'une voix bouleversée. Seul, j'étais avec toi, nous restions ensemble. Le premier voyage d'après-guerre : tu n'as pas le droit de le faire avec une autre. (Beauvoir de, Les Mandarins)

La contrefactualité s’applique à un événement non pas passé mais futur: le voyage au Portugal dont il est question est à venir... Vertige ? Même pas : le tour contrefactuel apparaît un peu plus encore comme un tour hypothétique, dans la mesure où le conditionnel passé peut lui aussi, dans ce type de tour, porter sur un événement futur :

(38) - Qu'est-ce que je voulais en venant ici dans ce cas ? demanda Drago, un léger reproche dans la voix.

- La même chose que tu aurais voulu si tu étais venu demain matin. (Internet)

\section{Références bibliographiques}

Berthonneau A.-M. et Kleiber G., 2003, «Un imparfait de plus... et le train déraillait », Cahiers Chronos 11, 1-24.

Berthonneau A.-M. et Kleiber G., 2006, «Sur l'imparfait contrefactuel », Travaux de linguistique $\mathrm{N}^{\circ} 53,7-65$.

Barceló G. J. et Bres J. 2006, Les temps de l’indicatif, Paris : Ophrys.

Bres J., 2005, L’imparfait dit narratif, Paris : CNRS-Editions.

Bres J., 2006, «Encore un peu, et l'imparfait était un mode...». L'imparfait et la valeur modale de contrefactualité », Cahiers de praxématique 46, 149-176.

Bres J., 2007, «Sémantique de l'imparfait : dépasser l’aporie de la poule aspectuelle et de l'œuf anaphorique ? Eléments pour avancer », Cahiers Chronos 16, 23-46..

Bres J., 2008, « Les plus désaccordés sont les temps les plus beaux... La question des emplois « stylistiques » des temps verbaux de l'indicatif en français », Cahiers chronos, à paraître.

Bres J., 2009, "Alors comme ça le conditionnel serait polyphonique... », Colloque La question polyphonique, Metz-Luxembourg, 8-10 septembre, à paraître.

Dowty D., "The effects of Aspectual Class on the Temporal Structure of Discourse: Semantics or Pragmatics”, Linguistics and Philosophy 9.1, 37-62.

Gosselin L., 1998, "Le "paradoxe imperfectif”, ou la disjonction entre assertion et predication ", in Forsgren M., Jonasson K. et Kronning H., Prédication, assertion, information, Uppsala : Studia Romanica Upsaliensia 56, 211-220.

Guillaume G, 1929/1970, Temps et verbe, Paris : Champion.

Guillaume G., 1971. Leçons de linguistique 1948-1949, Série A, tome I, Québec : Presses de l'université Laval et Paris : Klincksieck.

Kleiber G., 2003, «Entre les deux mon cœur balance, ou l'imparfait entre aspect et anaphore ", Langue française 138, 8-19.

Kleiber G., 2006, «L'imparfait des deux rives: entre aspect et anaphore », in Bertrand D. et Fontanille J. (éds), Régimes sémiotiques de la temporalité, PUF, 91-136.

Kleiber G. et Berthonneau A.-M., 2002, "L'imparfait contrefactuel : d'une explication l'autre », in Anis J., Eskénazi A., Jeandillou J. -F., Le signe et la lettre, Hommage à Michel Arrivé, Paris : L’Harmattan, 321-336. 
Labov W., 1978, «La transformation du vécu à travers la syntaxe narrative », dans Le parler ordinaire I, Paris : Minuit, 289-355.

Riegel M., Pellat J.-Ch. et Rioul R., 1994, Grammaire méthodique du français, Paris : PUF. Wilmet M., 1997 / 2003, Grammaire critique du français, Paris : Hachette, Louvain-la

Neuve : Duculot. 